Document downloaded from:

http://hdl.handle.net/10251/104131

This paper must be cited as:

Pastor, JV.; García-Oliver, JM.; García Martínez, A.; Zhong, W.; Mico Reche, C.; Xuan, T. (2017). An Experimental Study on Diesel Spray Injection into a Non-Quiescent Chamber. SAE International Journal of Fuel and Lubricants. 10(2):1-13. doi:10.4271/2017-01-0850

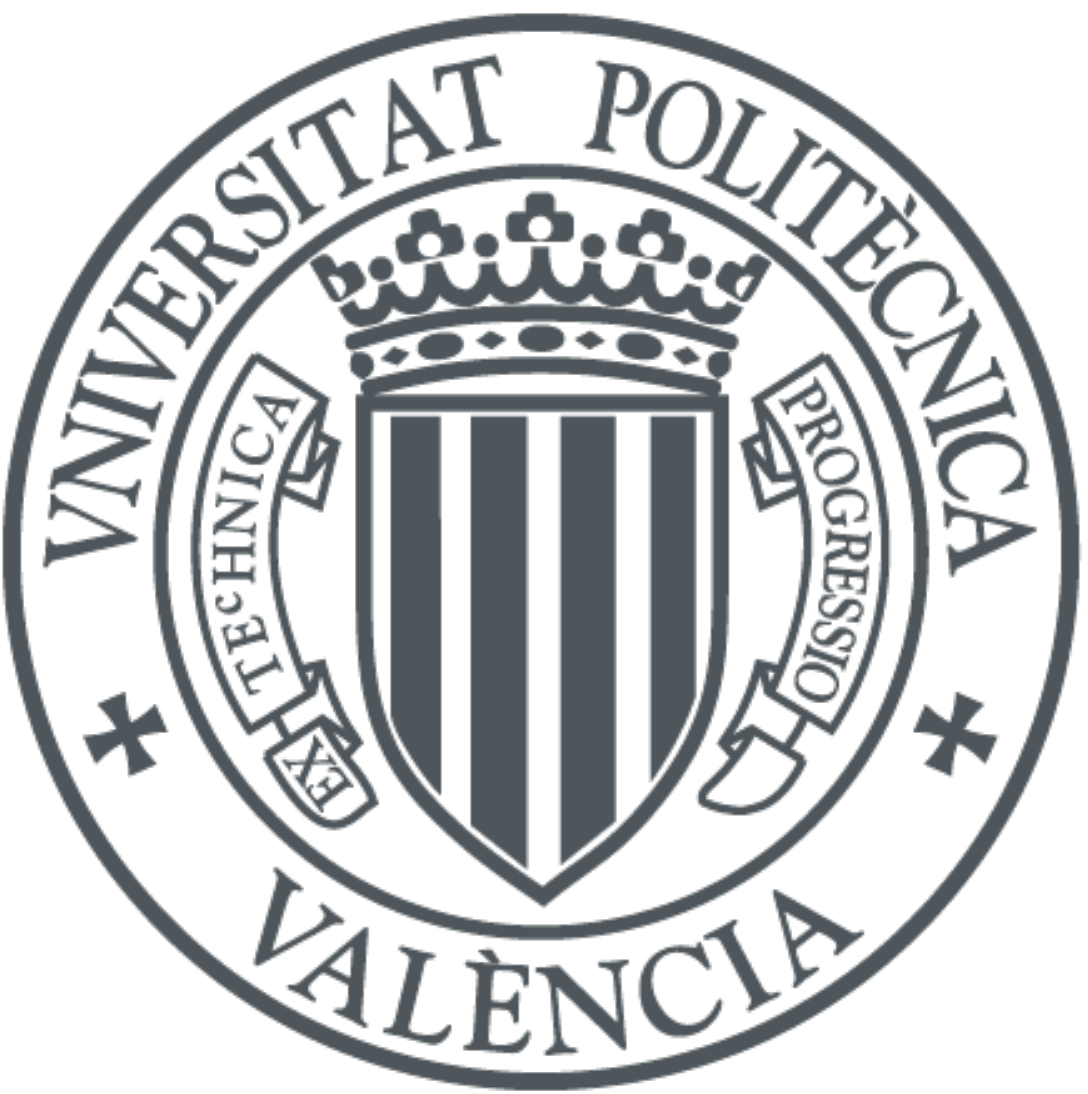

The final publication is available at

http://doi.org/10.4271/2017-01-0850

Copyright SAE International

Additional Information 


\title{
An experimental study on Diesel spray injection into a non-quiescent chamber
}

\author{
Author, co-author (Do NOT enter this information. It will be pulled from participant tab \\ in MyTechZone) \\ Affiliation (Do NOT enter this information. It will be pulled from participant tab in MyTechZone)
}

\author{
Copyright $\odot 2017$ SAE International
}

\begin{abstract}
Visualization of single-hole nozzles into quiescent ambient has been used extensively in the literature to characterize spray mixing and combustion. However in-cylinder flow may have some meaningful impact on the spray evolution. In the present work, visualization of direct diesel injection spray under both non-reacting and reacting operating conditions was conducted in an optically accessible two-stroke engine equipped with a singlehole injector. Two different high-speed imaging techniques, Schlieren and UV-Light Absorption, were applied here to quantify vapor penetration for non-reacting spray. Meanwhile, Mie-scattering was used to measure the liquid length. As for reacting conditions, Schlieren and $\mathrm{OH}^{*}$ chemiluminescence were simultaneously applied to obtain the spray tip penetration and flame lift-off length under the same TDC density and temperature. Additionally, PIV was used to characterize in-cylinder flow motion. Results were compared with those from the Engine Combustion Network database obtained under quiescent ambient conditions in a high pressure high temperature vessel. Because of the air flow induced by piston movement, in-cylinder conditions in the two-stroke engine during the spray injection are highly unsteady, which has a significant impact on the spray development and interference on the spray visualization. From the comparison with quiescent data from the Engine Combustion Network, air flow induced by piston movement was found to slow down tip penetration. Moreover, both ignition delay and lift-off length under unsteady flow conditions show less sensitivity with ambient temperature than that of quasi-steady conditions.
\end{abstract}

\section{Introduction}

Fuel-air mixing plays a significant role in combustion and emission process in diesel engine. Better understanding on the effect of different operating conditions on the mixing between spray and ambient gas will help improve the efficiency and meet the increasingly stringent emission regulations.

For the last decades, thanks to the development of all kinds of optical techniques, many investigations have been done to study some quantitative spray and flame parameters during the fuel-air mixing and combustion process. Injection usually takes place into a high pressure high temperature vessel at constant volume or pressure, where the air-flow velocity is quite low compared with that of the injected spray, so the ambient conditions can be considered as quiescent. Plenty of experiments are available in the literature either from constant-volume vessels or constant pressure ones. Detailed analysis on the effects of different operating conditions (gas density, ambient temperature, oxygen concentration) and injection pressure on the vapor penetration[1,2], liquid length[3,4], flame lift-off length[5,6], ignition delay[7,8] or even reacting spray dynamics have been investigated [9-11]. Thanks to these efforts, the mechanism of the mixing and combustion process of quasi-steady diesel sprays is becoming clear. On the other hand, spray development in the actual engine very often occurs under non-quiescent conditions. A lot of investigations on these spray and combustion parameters mentioned above have been done in different optical engines [12-15] with single-hole injectors. What's more, considering that multi-spray injectors are usually applied in real diesel engines, the influence of jet-to-jet interactions on combustion and soot formation have also

Page 1 of 12 been investigated by some researchers [16,17]. However, the information related to the influence of air flow brought about by piston movement on spray and combustion development is still limited. The characterization of swirl flow structure in a light-duty optical diesel engine was studied in $[18,19]$ by Particle Image Velocimetry (PIV) coupling CFD tools. Authors concluded that piston geometry effect on the flow asymmetry might be more responsible than the intake flow effect. In [20], a high fluctuation of lift-off length was found for measurements in the engine applied in the present paper. Cycle-to-cycle scattering of air flow may be one of the main reasons for this behaviour.

In the present work, experiments were performed in an adapted twostroke optical diesel engine. High-speed Schlieren imaging was applied to detect the complete boundary of the non-reacting and reacting spray. However, a limit was found for this technique when measuring the non-reacting spray cases because of the strong air movement. As a consequence, a newly developed Ultraviolet Light Absorption (UV-LA) technique was also applied here for the vapor penetration measurement. Mie-scattering and $\mathrm{OH}^{*}$

chemiluminescence were used to measure the liquid length and flame lift-off length (LOL) for non-reacting and reacting sprays separately. Additionally, PIV was used to characterize in-cylinder flow motion. Measurements were performed with single-hole nozzles, and the analysis will show that the interaction of the spray with a nonquiescent medium cannot be neglected.

Including the present introduction, this document is made up of four sections. The next section (Experimental Setup and Methodology) gives a detailed description about the experimental facility, different 
optical techniques, and the test plan which was investigated in this paper. In the third section (Results and Discussion), the

characterization of in-cylinder airflow is presented. Next, the effects of airflow on both non-reacting and reacting spray are elaborated.

Meanwhile, all these spray and combustion parameters are compared with experimental results from the Engine Combustion Network (ECN) [21] under quiescent conditions. The last section of the paper (Conclusions) summarizes some of the most important conclusions of this investigation.

\section{Experimental Setup and Methodology}

\section{Test rig}

An optically accessible single cylinder two-stroke engine with threeliter displacement, 15.6:1 compression ratio and low rotational speed of $500 \mathrm{rpm}$ has been used for these experiments, which is described in detail in [22]. A cylindrical combustion chamber is designed with a diameter of $45 \mathrm{~mm}$. This chamber has one upper access for the fuel injector, and four lateral orthogonal accesses. One of them is used for the pressure transducer whereas the other three are equipped with optical windows with geometrical dimensions of $88 \mathrm{x}$ $37 \mathrm{~mm}$ and $28 \mathrm{~mm}$ thick. The Cross-sectional view of cylinder head is shown in Figure 1.During engine operation, the block temperature is controlled by an external heating-cooling system. The intake air temperature and pressure are controlled by electrical resistors and an air compressor respectively. An injection takes place every 30 cycles, which guarantees that there is no remaining residual gas from previous combustion cycles and the ambient conditions in the chamber are kept constant between consecutive repetitions. A commercial common-rail piezoelectric injector equipped with a single-hole nozzle $0.082 \mathrm{~mm}$ in diameter was used in this study.
Thanks to the adequate cooling of the injector holder and the low injection frequency during operation, the nozzle tip temperature can be considered constant.

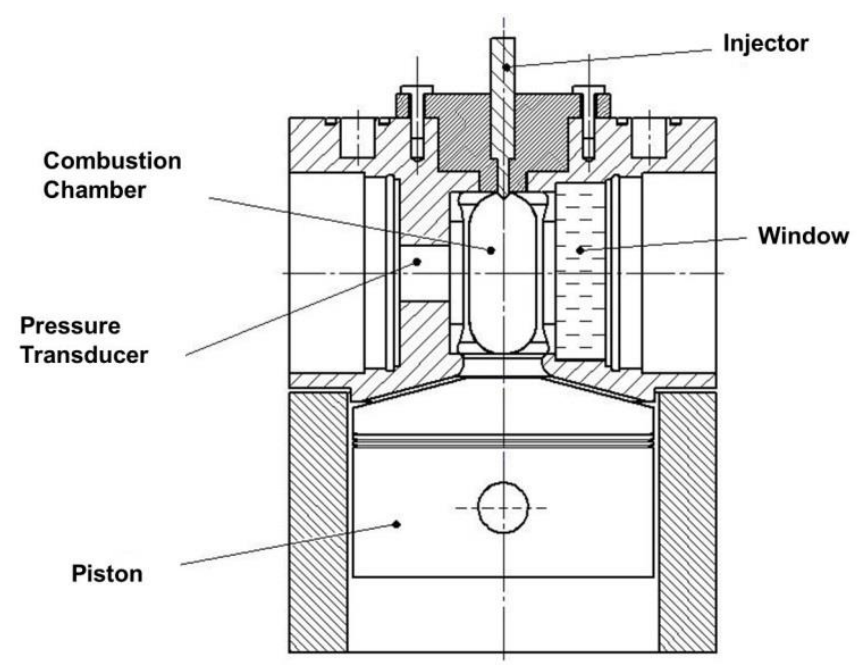

Figure 1. Cross-sectional view of cylinder head

\section{Optical techniques}

As mentioned above, high-speed Schlieren and UV-LA imaging were applied here for measuring the vapor penetration under non-reacting conditions. The schematic of optical setup for non-reacting spray tests is shown in Figure 2 (a) and Figure 2 (b). As for the reacting spray tests, Schlieren and $\mathrm{OH}^{*}$ chemiluminescence were applied simultaneously, as shown in Figure 2 (c).

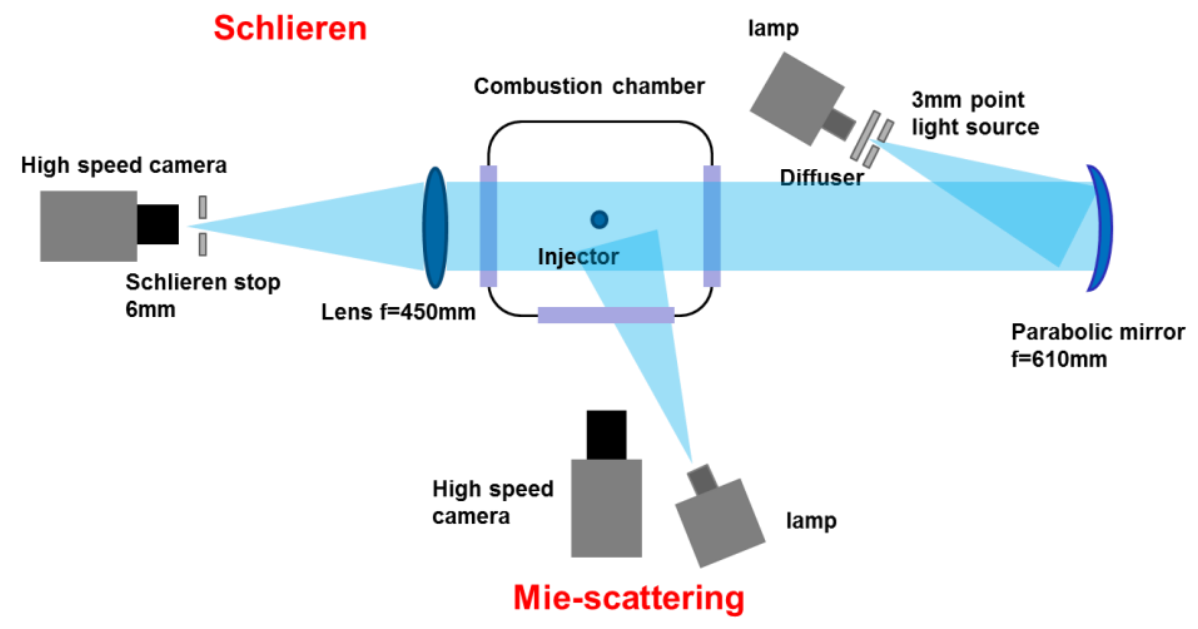

(a)

Page 2 of 12 


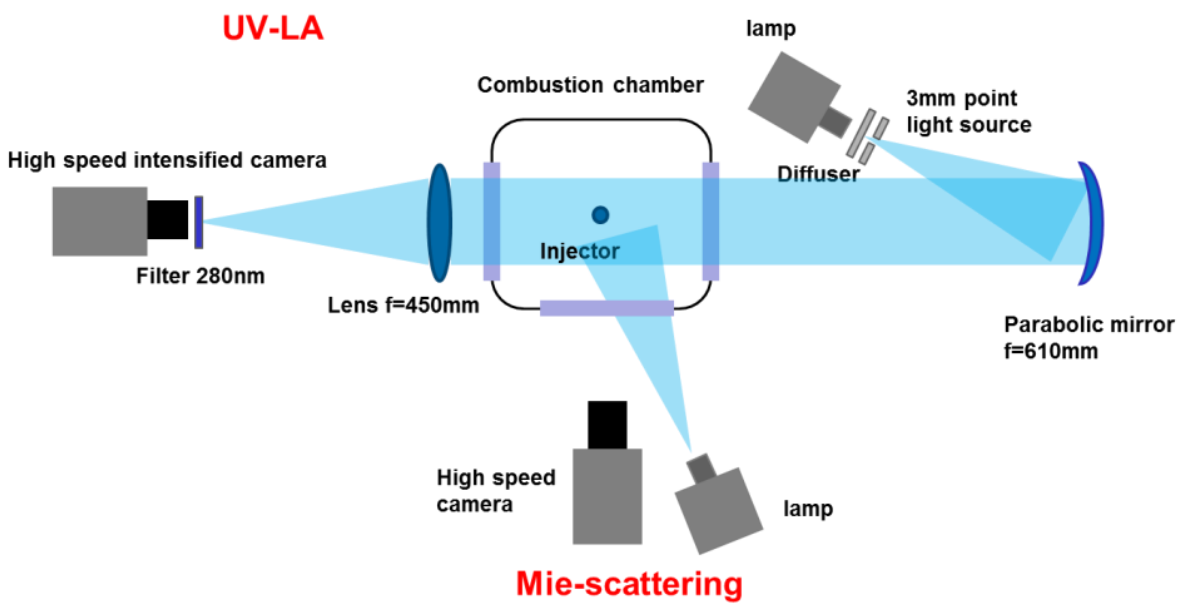

(b)

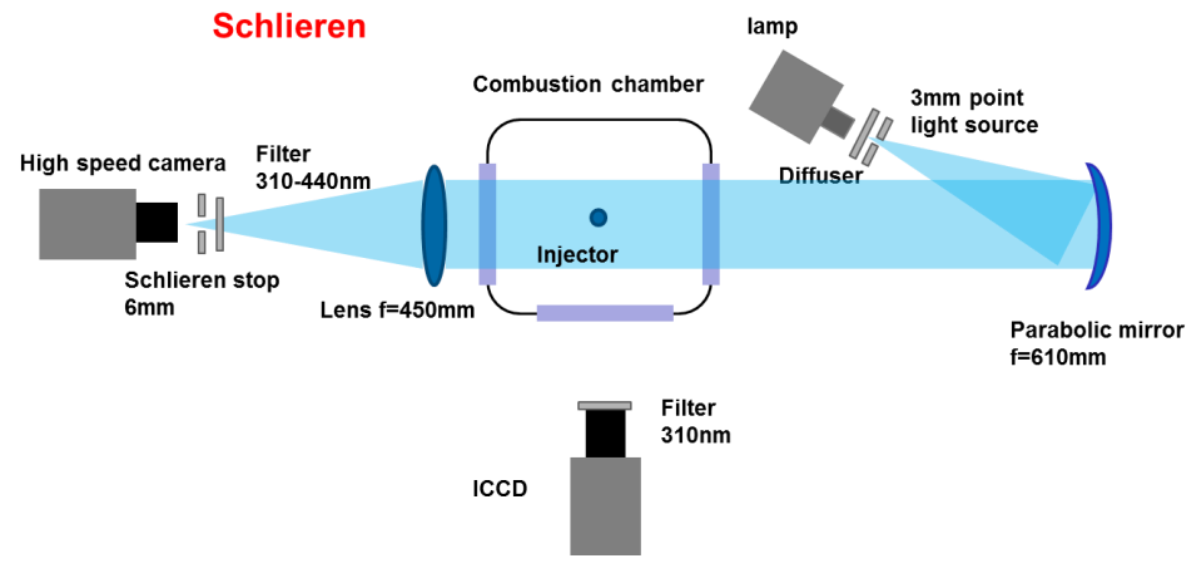

$\mathrm{OH}^{\star}$ Chemiluminescence

(c)

Figure 2. Optical setup (a) Schlieren and Mie-scattering (b) UV-LA and Mie-scattering (c) Reacting test

\section{Schlieren imaging}

Schlieren imaging is a valuable technique for identifying density gradients, from which the spray area can be derived because of the density difference between vaporized fuel and ambient gases. The technique is based on the deviation suffered by a light beam, due to its refraction when crossing from one media to another, with different refractive index. This effect is known as beam steering. If a continuous non-homogeneous media is considered, the variation of the refractive index is represented by its gradient. Thus, it can be demonstrated that the deviation suffered by a light beam when traversing this media is proportional to the refractive index gradients within it [23]. Thanks to this phenomenon, the diesel spray has usually been able to be distinguished easily against the background light. In addition, besides spray tip penetration, ignition delay was also obtained from Schlieren images based on the analysis on the total intensity increment within the spray between each two following images. The detail of this processing methodology can be found in [24], where the corresponding time of the peak of the total intensity increment was defined as ignition delay and it was also validated by broadband chemiluminescence technique.

The actual Schlieren optical setup is shown in Figure 2 (a). A diffused point light obtained from a Xenon arc lamp was collimated by a parabolic mirror $(\mathrm{f}=610 \mathrm{~mm})$ which directs it through the combustion chamber. A spherical lens $(\mathrm{f}=450 \mathrm{~mm})$ was placed on the other side of the chamber to focus the light onto a so-called Fourier plane. A diaphram $(6 \mathrm{~mm})$ was used here as the Schlieren stop so that the light is later collected by the high speed-CMOS camera (Vision Research Phatron SA-5) running at 30,000 frames per second (fps) with a spatial resolution $6.8 \mathrm{pixel} / \mathrm{mm}$. As for the reacting spray measurement, in order to eliminate soot radiation effect, a bandpass filter (310-440nm) was placed in front of the Schlieren stop (as shown in Figure 2 (b)) and the shutter time is reduced to $0.37 \mu \mathrm{s}$ compared with $9.85 \mu$ s for non-reacting spray, while the other settings were kept the same with non-reacting one. In typical Schlieren setups, two parabolic mirrors are usually used off-axis at equal and opposite angles so that the off-axis aberrations eliminate each other. In this work, only one parabolic mirror was used, but the large focal

Page 3 of 12 
length of the mirror has reduced the comma and astigmatism effects on the images.

\section{UV-Ligth Absorption (UV-LA)}

Besides Schlieren imaging, the vapor penetration of non-reacting spray was also measured by a developed UV-LA technique which is based on the ultraviolet (UV) light attenuation by the vapor phase $[14,25]$. Because of this absorption for UV light, the intensity within the spray is much lower than that of the background gas area. As a consequence, the spray boundary was identified easily. The vapor optical thickness, KL, can be obtained according to the well-known Beer-Lambert law.

$$
\log \left(\frac{I_{0}}{I_{t}}\right)=K L
$$

where $\mathrm{I}_{0}$ is the incident radiation, as obtained from images before start of injection, and $\mathrm{I}_{\mathrm{t}}$ is the intensity of transmitted light. $\mathrm{K}$ is the dimensional extinction coefficient and $\mathrm{L}$ is the path length of the light beam through the vapor phase. A fixed threshold of $20 \%$ the maximum KL value from KL images at each time position was applied here to distinguish the spray vapor phase and the background air.

The optical setup is quite similar with that of Schlieren imaging, as shown in Figure 2 (b). A continuous broadband 1000 W Xenon Arc lamp was used here as the light source to create the desired UV light. On the collection side, an intensified high speed camera Photron I2 coupled to an interference filter (centered at $280 \mathrm{~nm}$, with $10 \mathrm{~nm}$ FWHM) was applied. Other settings were kept same as that of Schlieren. It must be noted that the UV light was also collimated by the parabolic mirror. The images were recorded at a speed of 8000 fps and a spatial resolution $8 \mathrm{pixel} / \mathrm{mm}$.

\section{$\mathrm{OH}^{*}$ chemiluminescence}

Lift-off length was measured by recording the signal from $\mathrm{OH}^{*}$ chemiluminescence, being a marker of the diffusion flame limits. A schematic of the actual optical setup is also shown in Figure 2(c). An Andor Solis iStar ICCD intensified camera equipped with a $100 \mathrm{~mm}$ focal length $\mathrm{f} / 2 \mathrm{UV}$ objective and a $310 \mathrm{~nm}$ interference filter (FWHM $=10 \mathrm{~nm}$ ) was used. Only one image per injection event was recorded from $4 \mathrm{~ms}$ to $5 \mathrm{~ms}$ after start of energizing (ASOE) with a $\mathrm{pixel} / \mathrm{mm}$ ratio of 10.9 . One example of $\mathrm{OH}^{*}$ radiation image is shown in Figure 3.

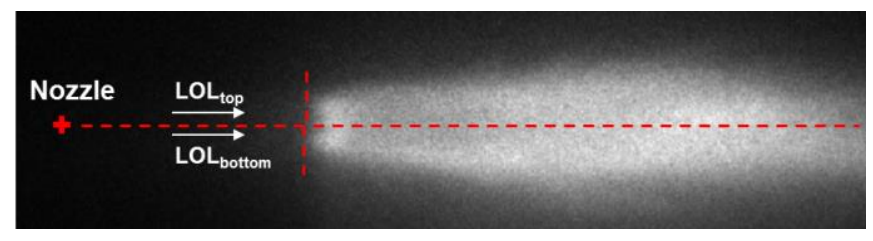

Figure 3. Schematic of LOL

Page 4 of 12

\section{Particle Image Velocimetry (PIV)}

To better understand the gas flow effects on spray behavior, it was decided to characterize the airflow movement within the combustion chamber under motored conditions by means of PIV. This technique is based on measuring the displacement of a cloud of particles between two consecutive images. For this purpose, a pulsed laser is usually employed to create an intense light sheet that illuminates the particles. The scattered light is registered by a CCD camera. A more detailed description of this technique can be found in [26] [27]. The optical set-up is depicted in Figure 4. A double-head pulsed Nd:YAG (2 lamps) laser was utilized to generate two pulses at $532 \mathrm{~nm}$. The laser beam was guided to the combustion chamber by means of a laser line mirror, while a cylindrical lens (-10 $\mathrm{mm}$ focal length) was used to transform the beam into a laser sheet with a frequency that can range from $0-15 \mathrm{~Hz}$. A spherical lens (1000 $\mathrm{mm}$ focal length) was utilized to minimize the beam waist at the region of interest. The light scattered by particles was registered by a CCD camera, working under "frame-straddling" mode [28].The straddle frequency is 7.5 pairs/s.

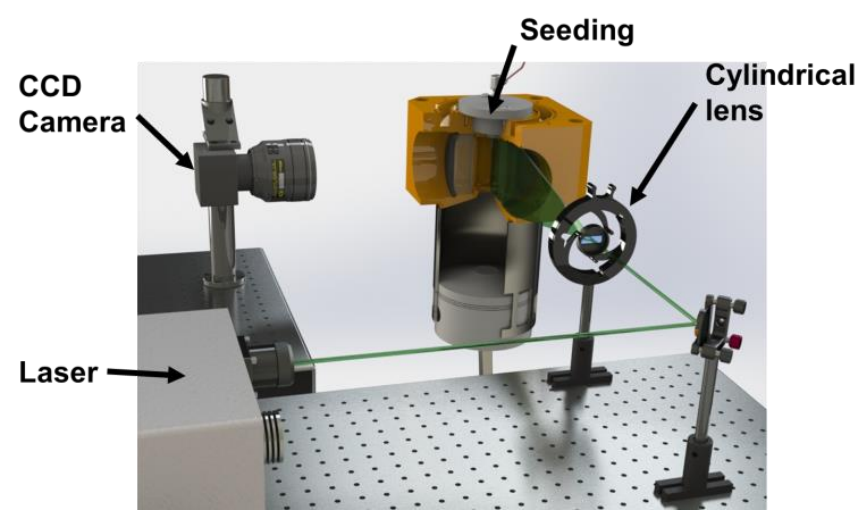

Figure 4. Schematic of PIV optical setup

Measurements were performed, under motored engine conditions, i.e. only the piston-induced flow was measured. Thermodynamic conditions at TDC correspond to $\rho_{a}=19.27 \mathrm{~kg} / \mathrm{m} 3$ and $T_{a}=760 \mathrm{~K}$. Incylinder air was seeded with injection rate test fluid injected directly into the combustion chamber through the same injector as in spray investigations. In this case, a long injection is performed just after the Exhaust Vent Closure (EVC), which occurs at $-90^{\circ}$ ATDC. Because of the low volatility of the test fluid, as well as the reduced TDC temperature compared to the later spray injection studies (check Table 1), the oil droplets did not vaporize. Injection pressure was set to $1000 \mathrm{bar}$, so a good agreement between uniformity and quality of the images was obtained. Taking into account the range of velocities existing in the combustion chamber for the conditions chosen, the two laser pulses were separated $10 \mu \mathrm{s}$. Six different planes within the combustion chamber were measured. In Figure 5, a scheme of the distribution of the region of interest is shown. Two perpendicular planes intersecting on the spray axis (labelled as C and CC respectively), were chosen together with the corresponding parallel planes separated $10 \mathrm{~mm}$ on both sides of the combustion chamber, which are labelled as L (left), R (right), F (front) and B (back), respectively. Therefore, it was possible to determine not only the flow pattern, but also any possible asymmetry. Planes F-CC-B are within the line of sight of the later arrangement for Schlieren and UV-LAS visualization, and will be used for subsequent analysis. Measurements were performed from -10 to $15^{\circ}$, with $1^{\circ}$ increments. For each plane and instant, 75 cycles were recorded. It has to be 
noted that only one velocity map was recorded per engine cycle. To get an estimation of a theoretical limit to velocity, the maximum piston speed has been compared to the measured PIV field. If an incompressible steady flow is assumed, the following equation would give an estimation of the maximum chamber velocity:

$$
V_{\text {chamber }}=V_{\text {piston }} \cdot\left(A_{\text {piston }} / A_{\text {chamber }}\right)
$$

Giving values to the maximum piston speed $(\sim 4.6 \mathrm{~m} / \mathrm{s})$ and areas, the maximum chamber velocity is $51 \mathrm{~m} / \mathrm{s}$, which is far above the maximum PIV measured value $(20 \mathrm{~m} / \mathrm{s})$, as will be shown in later section.

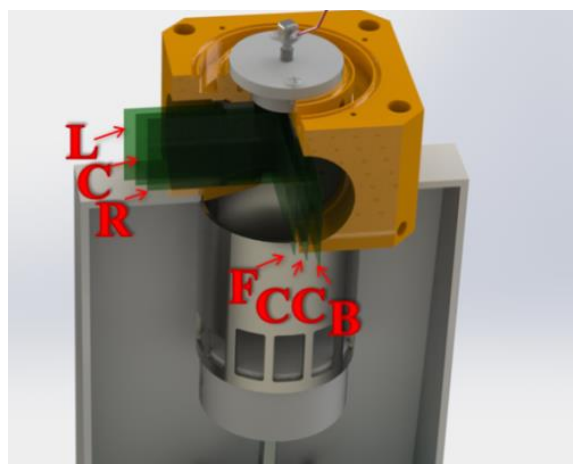

\section{Test Matrix}

The operating conditions forming the test matrix are summarized in Table 1, where in-cylinder conditions are parametrized in terms of TDC temperature $T_{a}$ and density $\rho_{a}$. The operating point NO (nominal condition, $\mathrm{T}_{\mathrm{a}}=870 \mathrm{~K}, \rho_{\mathrm{a}}=22.8 \mathrm{~kg} / \mathrm{m} 3$ ) was chosen as the baseline condition. Parametric variations in injection parameters (injection pressure) and thermodynamic conditions within the chamber at TDC (ambient temperature and ambient density) were performed both under non-reacting (in-cylinder mixture contained pure nitrogen) and reacting conditions (injection into ambient air, with $21 \%$ oxygen (vol.)). The test matrix also includes a condition denoted as SA that reproduces TDC conditions similar to the socalled Spray-A condition from ECN, but at $21 \%$ oxygen volume fraction. This makes it possible to compare the evolution of sprays in the present combustion chamber with ECN data measured in a constant pressure chamber, where thermodynamic and flow conditions are nearly quiescent and steady. The PIV operating condition was presented in the last row of Table 1 .

A common rail single-hole injector fitted with a $82 \mu \mathrm{m}$ nozzle has been used in the experiments. The fuel used throughout these tests was n-Dodecane for all techniques except for the UV-LA imaging technique, where $80 \%$ of dodecane (in mass) was blended with a blend of additional components with high absorptivity for UV light. More information about this blend can be found in reference [27]. For all experiments performed within the present study, 30 injections have been recorded at each operating condition to reduce measurement uncertainties due to engine operating variability.

Figure 5. Scheme of the distribution of the PIV measuring planes.

Table 1 Experimental conditions

\begin{tabular}{cccccc}
\hline Operating point & $\mathbf{P}_{\text {inj }}[\mathrm{bar}]$ & $\begin{array}{c}\text { Air } \mathbf{T} \\
\left(\mathbf{T}_{\mathbf{a}}\right)[\mathbf{K}]\end{array}$ & $\begin{array}{c}\text { Air Density } \\
\left(\boldsymbol{\rho}_{\mathrm{a}}\right)[\mathrm{kg} / \mathbf{m} 3]\end{array}$ & $\begin{array}{c}\text { Oxygen } \\
{[\%]}\end{array}$ & COMMENTS \\
\hline NO & 1500 & $\mathbf{8 7 0}$ & $\mathbf{2 2 . 8}$ & $\mathbf{0 / 2 1}$ & Baseline \\
SA & $500 / 1000 / 1500$ & $\mathbf{9 0 0}$ & 22.8 & $0 / 21$ & ECN-Spray A \\
MT & 1500 & $\mathbf{8 3 0}$ & 22.8 & 21 & Medium T \\
LT & 1500 & $\mathbf{7 8 0}$ & 22.8 & 21 & Low T \\
LD & $\mathbf{1 5 0 0}$ & $\mathbf{8 7 0}$ & $\mathbf{1 5 . 2}$ & $\mathbf{0}$ & Low density \\
\hline \hline $\begin{array}{c}\text { PIV operating } \\
\text { condition }\end{array}$ & - & $\mathbf{7 6 0}$ & $\mathbf{1 9 . 2 7}$ & $\mathbf{0}$ & Motored condition \\
\hline
\end{tabular}


To determine the intake pressure and temperature values required to achieve the target TDC conditions, an accurate characterization of the engine has been performed. Thermodynamic conditions inside the cylinder have been calculated from measured pressure, using a firstlaw thermodynamic analysis. An example of the in-cylinder pressure and density temporal evolution during the injection event at NO condition is plotted in Figure 6. The injector was energized starting at $-6.35^{\circ}$ ATDC, while the actual injection starts at around $-5.35^{\circ}$ ATDC, to minimize piston-induced volume variations conditions during the injection event. The energizing time was set as $4 \mathrm{~ms}$, which results in an injection duration of $5 \mathrm{~ms}\left(15^{\circ}\right)$, approximately.

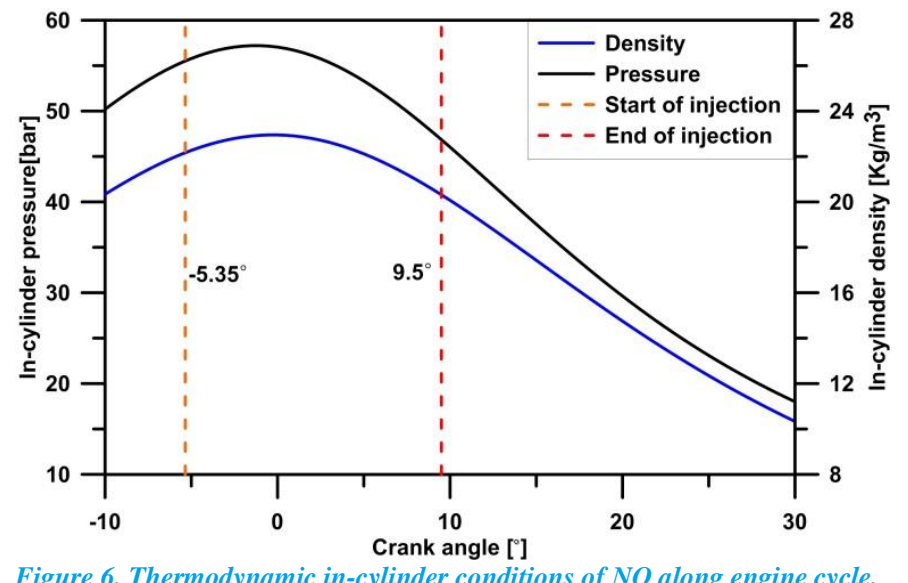

Figure 6. Thermodynamic in-cylinder conditions of NO along engine cycle.

\section{Results and Discussion}

\section{In-cylinder airflow under motored conditions}

Velocity fields of the airflow corresponding to the plane $\mathrm{CC}$ are shown in Figure 7. They have been obtained by averaging the velocity magnitude from 75 repetitions. One velocity map is shown every $2^{\circ}$ during the injection event (from $-5^{\circ}$ to $9^{\circ}$ ), as representative of the global behavior. The color map corresponds to the velocity magnitude (i.e. the modulus of the $2 \mathrm{D}$ projection of the velocity vector on the measurement plane), while the black lines represent the flow stream lines. At $-5^{\circ}$ ATDC the airflow is entering into the combustion chamber at more than $20 \mathrm{~m} / \mathrm{s}$, while at the upper part of the combustion chamber the speed is reduced to $5 \mathrm{~m} / \mathrm{s}$ approximately.
As piston approaches TDC position, velocity values decrease, but the high velocity region is observed to move closer to the nozzle and towards the left part of the combustion chamber. Near TDC this high velocity region vanishes drastically, as expected. However, a clockwise vortex appears on the right part of the combustion chamber. This flow structure seems to move towards the left of the combustion chamber and disappears as the piston moves down and the outlet flow speed increases.

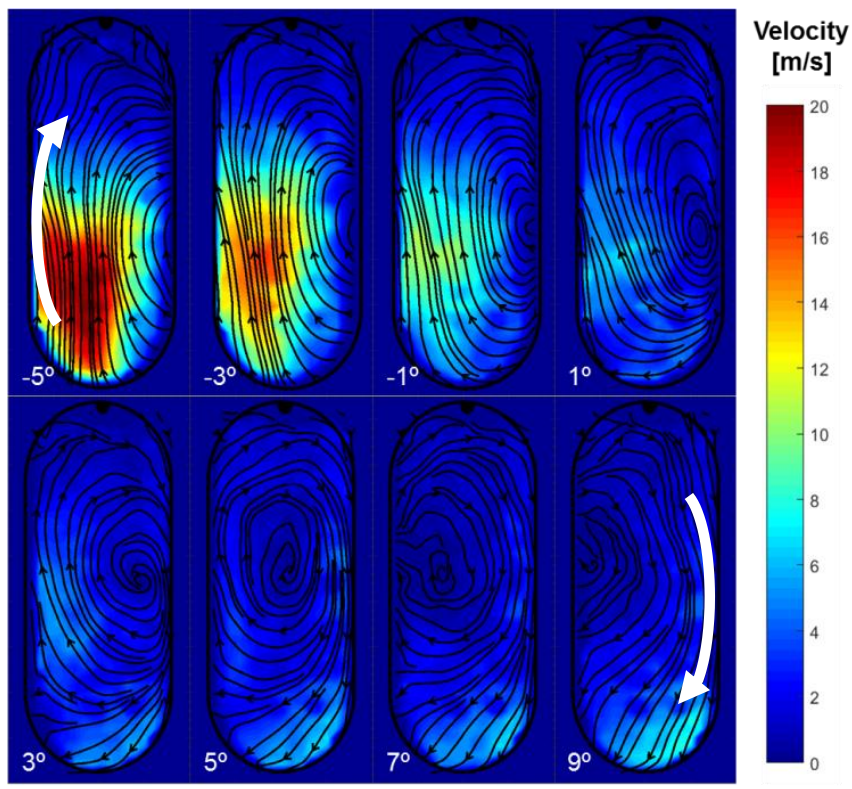

Figure 7 .Evolution of the average air flow velocity on plane CC. Color code corresponds to the velocity magnitude within the measurement plane.

A comparison of the averaged velocity fields among different planes at $-5^{\circ}$ ATDC is shown in Figure 8. Differences in magnitude and distributions can be observed among the different measuring planes which indicate the flow field is not symmetric within the combustion chamber. Velocities on L and B planes are usually higher than that on $\mathrm{R}$ and $\mathrm{F}$ respectively. The main consequence of these observations is that, the spray tip can be slowed down by the airflow and even displaced towards one side of the combustion chamber, as will be shown in later sections.

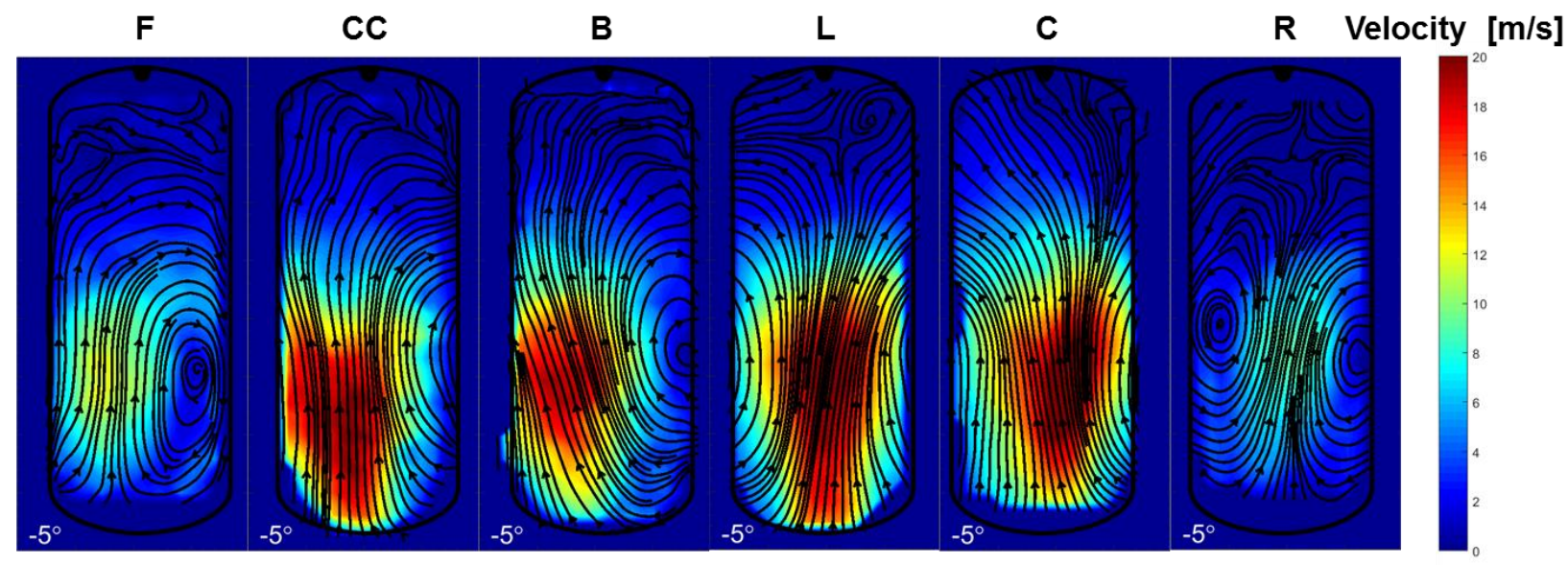

Figure 8. Air flow velocity on the different measuring planes at $-5^{\circ}$ ATDC. Color code corresponds to the velocity magnitude within the measurement plane. 


\section{Non-reacting spray analysis}

After the analysis of in-cylinder flow, Schlieren and Mie-scattering results are analyzed to describe non-reacting spray behaviour. The tip penetration of n-Dodecane at NO1500 and LD1500 are shown in Figure 9. In this figure as well as in the subsequent ones presented in this paper, the time is referred to the start of injection with the acronym ASOI (After Start of Injection) and all the plots represent the average value from 30 repetitions. The corresponding standard error (SE) with 95\% confidence level is also included. From Figure 9, the spray vapor of LD1500 is seen penetrating faster than that of NO1500 due to lower air entrainment because of the lower density, which is consistent with previous research [1]. However, these penetration curves tend to level-off after around $50 \mathrm{~mm}$, while the optical limitation is around $80 \mathrm{~mm}$. It looks as if these sprays 'stop' in the middle of combustion chamber. This phenomenon is considered as Schlieren limitation which is marked with the gray region.

Two examples of processed Schlieren images at two different time position are shown at the top of Figure 10. At start of injection $(1200 \mu \mathrm{s})$, fuel mass fraction within vapor phase is high, and accordingly density gradients are also important, which makes it possible for the processing routine to capture the spray boundary precisely, which is done based on a fixed threshold. However, with the increase of air entrainment further away from the nozzle, fuel mass fraction at spray tip decreases, and so do spray-induced density gradients. On the other hand, the velocity of the air at the other end of the chamber is much higher than that of upstream spray, as shown in previous PIV maps, which can also increase the air entrainment and make spray become thinner. In addition, the high flow velocity on that end of the chamber also creates a deflection of light rays, which produces a non-uniform time-variable background. As a result, the detection of the spray is not accurate enough, as it gets diluted into the noisy background. This can be seen in the Schlieren image at $3200 \mu \mathrm{s}$ in Figure 10, for which a spray almost as long as the previous image at $1200 \mu \mathrm{s}$ is detected. This explains the levelling-off effect shown on Schlieren penetration plots (Figure 9).

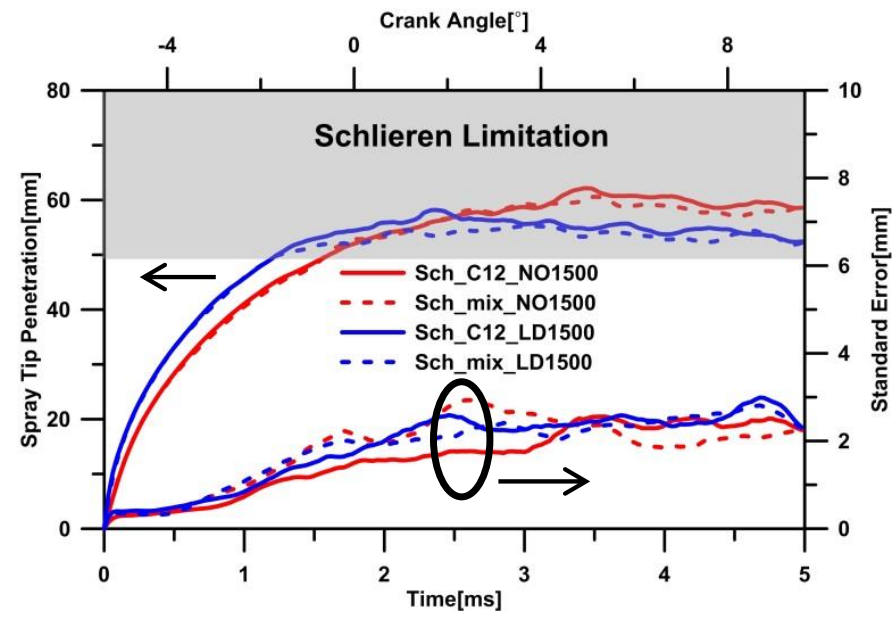

Figure 9. Spray tip penetration of n-Dodecane and mixture at NO1500 and LD1500 conditions as derived from high-speed Schlieren.

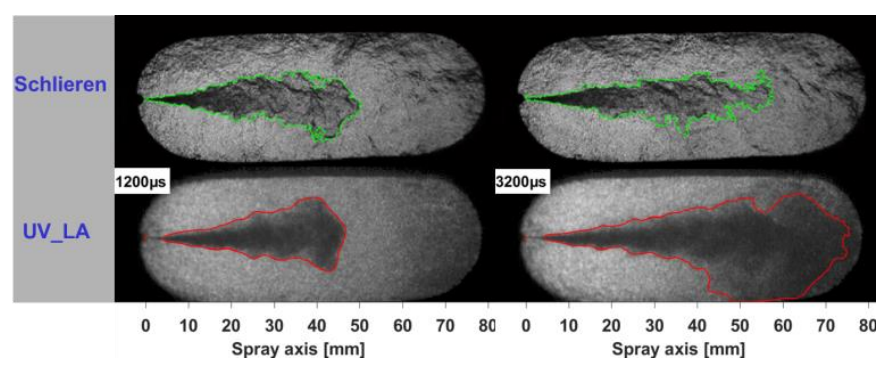

Figure 10. Comparison between Schlieren and UV-LA images at NO1500

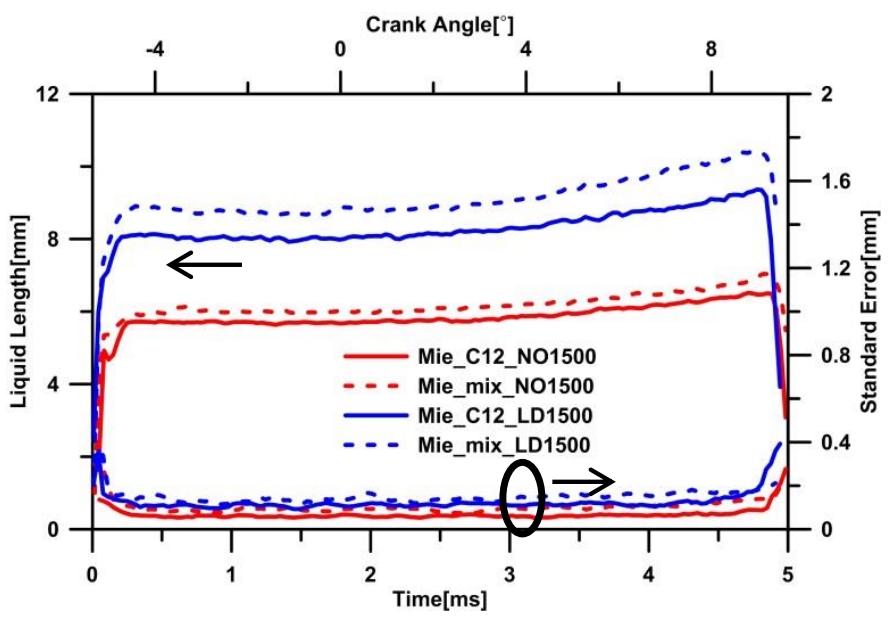

Figure 11. Liquid length of n-Dodecane and mixture at NO1500 and LD1500 conditions

To avoid the previous shortcomings from the Schlieren technique, UV-LA visualization was carried out, where the only absorbing media should be the fuel. As mentioned above, in order to get high absorptivity for UV light when doing UV-LA tests, $80 \%$ (in mass) n-Dodecane was blended with a highly-absorbing surrogate, detailed in [27]. The resulting fuel was investigated with Schlieren, Mie scattering and UV-LA techniques and compared against pure nDodecane in the first two techniques. Figure 9 and Figure 11 present a comparison of Schlieren-derived vapor penetration and Mie-derived liquid length of both fuels at NO1500 and LD1500 points.

Apparently, as shown in Figure 9, fuel effects on vapor penetration are negligible, because momentum flux, which is the main driver for penetration, does not depend on fuel properties when operating at constant injection pressure, which is coherent with previous research [29]. As a consequence, it can be concluded that vapor penetration of this mixture fuel can be applied to represent that of n-Dodecane. On the other hand, fuel composition is seen to have an influence on the liquid length, as shown in Figure 11. The liquid length of the mixture penetrates further because of the lower volatility, but maximum differences are less than $2 \mathrm{~mm}$, note that the absorbing fuel has still a large proportion of $n$-dodecane. Liquid length is also shown to slightly increase during the ending period of injection because of the ambient density decrease caused by the piston moving down from around $3^{\circ}$ ATDC. Summing up, the chosen fuel mixture will behave very similarly to $\mathrm{n}$-Dodecane as for vapor spray evolution.

Finally, Figure 12 shows tip penetration temporal evolution of mixture obtained both from Schlieren and UV-LA techniques at NO1500 and LD1500 points. It can be seen that the results from both techniques are consistent when the penetration is shorter than $50 \mathrm{~mm}$. After that, UV-LA is still able to capture the spray tip very well until 
the optical limit, which is also confirmed by the UV-LA image at $3500 \mu$ s in Figure 10. That is because ambient gas shows a quite low absorptivity for UV light compared with that of the vapor phase of the mixture spray and it is easy for the processing routine to identify the dark spray against the bright background. As a conclusion of this section, UV-LA performs better than Schlieren on capturing tip penetration for the non-reacting spray under non-quiescent conditions and the penetration value of $n$-Dodecane will be replaced with that of this mixture fuel for further analysis because of the negligible fuel effects.

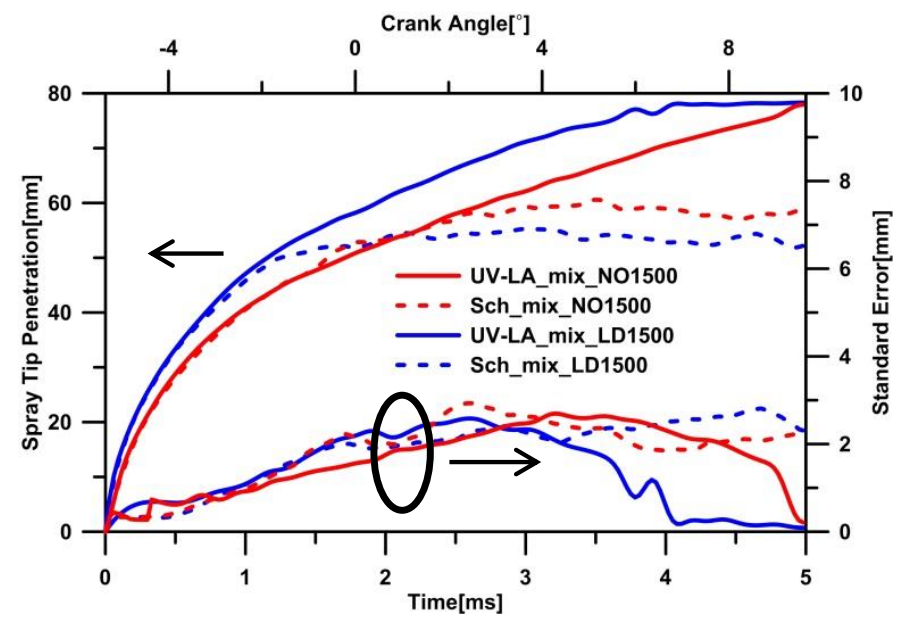

Figure 12. Schlieren and UV-LA comparison at NO1500 and LD1500 conditions

\section{Comparison with quiescent chamber results}

As a reference experiment under quiescent chamber conditions, the Spray A condition defined by ECN [20] has been chose, because it has the same density and temperature as defined at TDC for the SA operating point in the test matrix. The ECN database used here was obtained under quiescent conditions with n-Dodecane in a constant pressure vessel. A complete description of the facility is given in [30]. In addition, the standard Spray A injector was equipped with a single-hole nozzle with a diameter $90 \mu \mathrm{m}$.

On the other hand, for a mixing-controlled spray injected into a quiescent ambient, vapor tip penetration under constant injection and ambient boundary conditions can be described by the following equation:

$$
\mathrm{S}=\mathrm{k} \sqrt{\mathrm{u}_{0} \cdot \mathrm{d}_{0} \sqrt{\frac{\rho_{\mathrm{g}}}{\rho_{\mathrm{f}}}} \cdot \mathrm{t}}
$$

where $d_{0}$ is the orifice diameter, $u_{0}$ is injection velocity, $\rho_{\mathrm{g}}$ is ambient gas density, $\rho_{\mathrm{f}}$ is fuel density, $\mathrm{t}$ is time after start of injection and $\mathrm{k}$ is a proportionality constant that can be related to the air entrainment rate[31]. Compared to ECN data, the same fuel and injection pressure are used, and hence a similar injection velocity can be expected. Average chamber density in the engine for SA condition should also be similar to the nominal ECN one. Therefore, to enable a comparison between both sets of data, ECN results were scaled by orifice diameter as shown in the following equation:

$$
S_{\text {scale }}=S \cdot \sqrt{\frac{d_{0 E N G}}{d_{0 E C N}}}
$$

where $S_{\text {scale }}$ is the scaled penetration which will be used in the following comparison, $d_{0 E N G}$ and $d_{0 E C N}$ are the nozzle orifice diameter used in the optical engine and ECN tests, respectively.

Figure 13 shows the temporal evolution of tip penetration under SA non-reacting conditions for three injection pressures from both facilities. It has to be reminded that ECN vapor penetration was processed from Schlieren images, while engine data was obtained from UV-LA tests. It can be seen that, at start of injection, the vapor penetration from the engine is consistent with that from ECN. However, after around $40 \mathrm{~mm}$, divergence between both sets of data appears, and becomes larger with time. Although, the density drop due to piston motion might contribute to an increase on penetration velocity, the density difference from TDC to the end of injection is just around $2.5 \mathrm{~kg} / \mathrm{m} 3$, thus, its influence would be considered negligible. On the contrary, the gas flow was considered to play the main role.

One example of the temporal evolution of spray shape obtained from UV-LA at SA1500 condition is shown in Figure 14. The injection time and corresponding crank angle are marked on the top left and top right of this figure respectively. It can be seen, at early stage of injection $\left(-3.25^{\circ} \mathrm{ATDC}\right)$, the spray shape is cone-like, which is same as that under quiescent condition. This is because the spray is still far away from the window bottom and the velocity of ambient gas in that zone is quite low compared with the spray velocity, as shown in PIV images. However, from $2^{\circ}$ ATDC to $5^{\circ}$ ATDC, the spray geometry is much more complicated than a cone shape. First, the spray tip part becomes flat and then, some part at spray side is moving back. The PIV-derived flow streamlines are overlaid onto the UV-LAS images. It must be noted that PIV results were obtained under different incylinder conditions, to avoid evaporation of seeding test fluid. However, in a first approximation, the flow can be assumed to be the same due to the operation of the engine at the same rotational speed. A strong airflow was found at the bottom side moving in opposite direction towards the spray, with a clockwise vortex. The spray can be seen to follow such a flow pattern, which results in a much wider non-symmetrical spray tip.

In addition, standard error of penetration is also detecting this airflow effect, as shown in Figure 13. SE of engine measurements shows a small value at start of injection, very similar to the $\mathrm{ECN}$ ones for a quiescent ambient. By $1.5 \mathrm{~ms} \mathrm{SE}$ in the engine rises clearly above ECN results, which approximately coincides in time with the departure of the average penetration plots. Note that engine measurements are carried out with a much larger sample size (30 injections) than ECN ones. Therefore, air flow effects are observed in terms of both a slow-down of the average spray penetration, and also of an increase of shot-to-shot scattering. On the other hand, the lower injection pressure case also included in the plot shows a higher SE during the later phase of injection, which may be caused by the lower momentum within the spray at this lower injection pressure, which makes it more prone to disturbances from the air flow. 


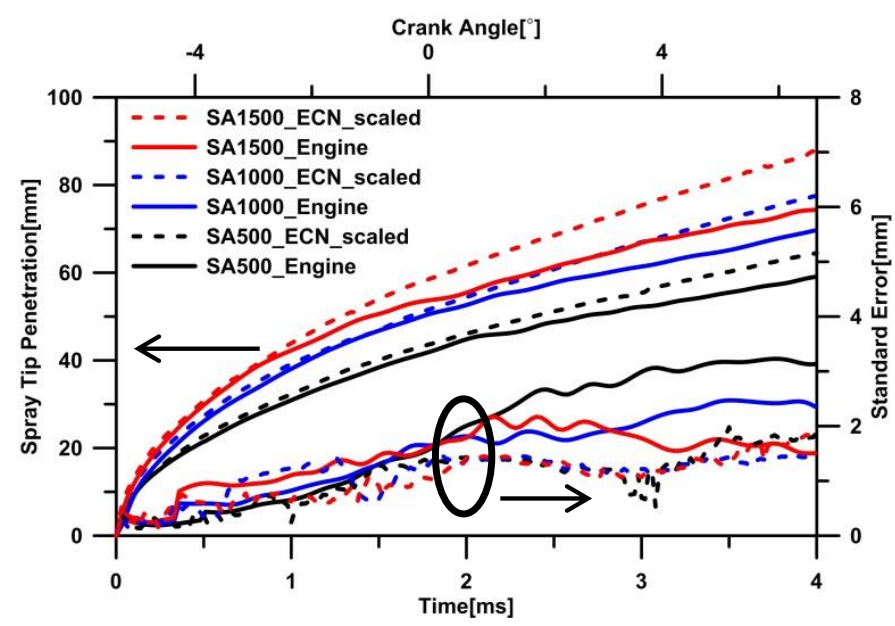

Figure 13. Comparison of vapor penetration with ECN data [11] and corresponding standard error of the mean under non-reacting conditions

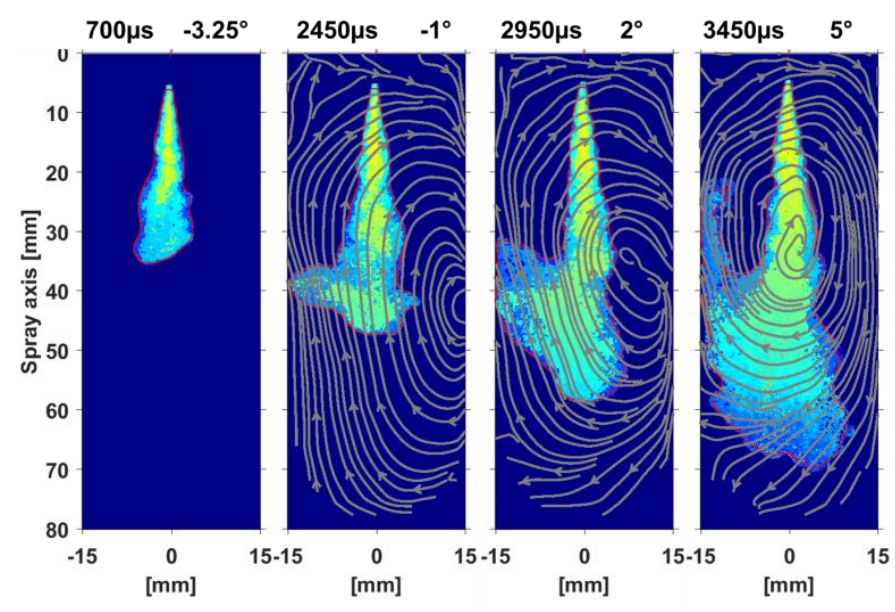

Figure 14. Superposition of PIV-derived streamlines and spray evolution from UV-LA processed images at SA1500 under non-reacting condition. Top labels indicate time ASOI (left) and piston position in crankangle degrees (right)

\section{Reacting spray analysis}

Spray tip penetration was measured with high-speed Schlieren under reacting conditions, where the high refractive density gradient created by combustion gives a very intense Schlieren signal, without the shortcomings of the non-reacting cases. A sequence of images of reacting spray temporal evolution at SA1500 were shown on the right of Figure 15, meanwhile, the non-reacting ones from UV-LA at corresponding time were shown on the left as reference. At start of injection $(200 \mu \mathrm{s})$, there is still no combustion happening, thus, the spray behaves as under non-reacting conditions, and there is no expansion neither in axial nor in radial direction. At that time, airflow influence is still non-significant as spray is far away from the high velocity zone. Around the ignition timing $(700 \mu \mathrm{s})$, combustion leads to a fast radial expansion on the spray, which produces such a slowdown of the spray tip penetration that it is even shorter than the non-reacting one. From $1200 \mu$ s to $2200 \mu$ s (around -2 to $1^{\circ}$ ATDC), spray tip is entering into the high velocity field zone. It can be seen that the tip penetration of reacting spray is being kept shorter than the non-reacting one. One speculation is that the airflow influence on reacting tip penetration might be stronger than the non-reacting one owning to the lower density caused by combustion within spray.

Page 9 of 12

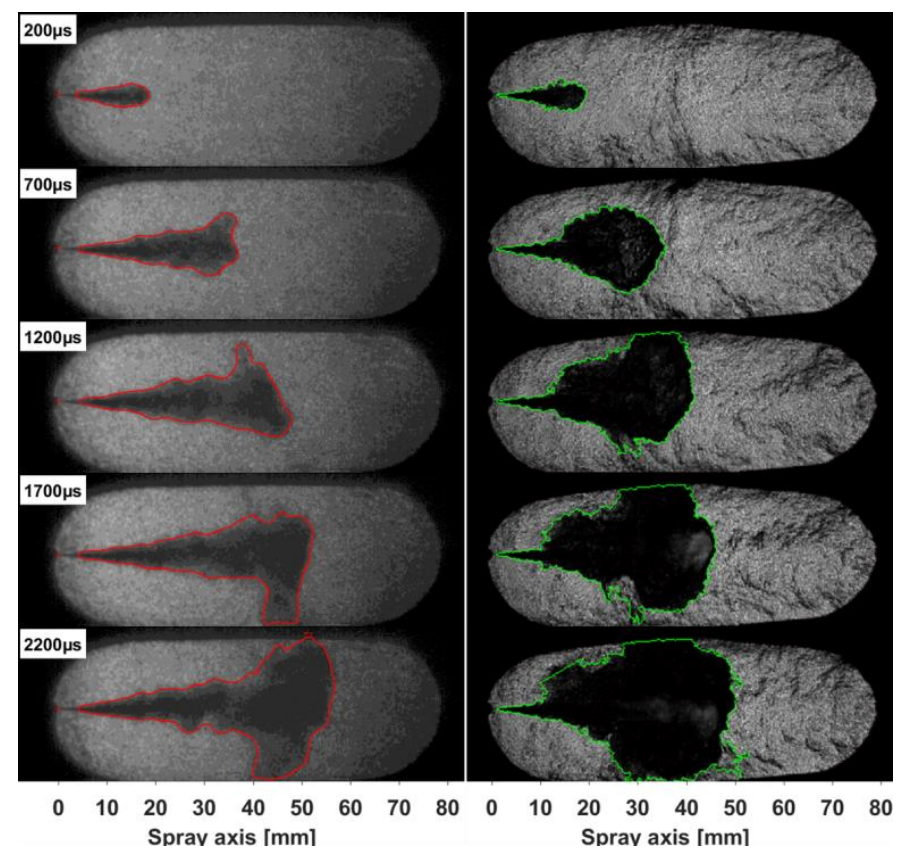

Figure 15. Non-reacting (left) and reacting (right) spray temporal evolution at SA1500 point

A comparison of reacting penetration with ECN data under SA1500 condition is shown in Figure 16, together with the non-reacting cases as a reference. Vertical solid and dashed lines represent ignition delay (ID) for engine and quiescent data, respectively. As shown in this figure, in both facilities reactive spray does not penetrate faster than the non-reacting one during the early stage of combustion. Based on momentum conservation, density drop due to combustion should result in a faster penetration of the spray. However, this is compensated by the strong initial increase in radial width. This stage is defined as 'stabilization phase' in reference [11]. After that, penetration difference of reacting spray between two facilities is larger than that of non-reacting spray. As for ECN case, reacting spray tip starts accelerating and penetrates faster than the nonreacting one after the radial expansion becoming stable [9-11]. One the other hand, a slower tip penetration in the reacting case compared to the non-reacting one is obtained in the present engine tests, as shown in Figure 16. This can be explained from different factors, such as aerodynamic forces, vortex and more air entrainment, brought by the airflow might be able to lead to a wider radial expansion compared with the non-reacting one, because of lower density within reacting spray. 


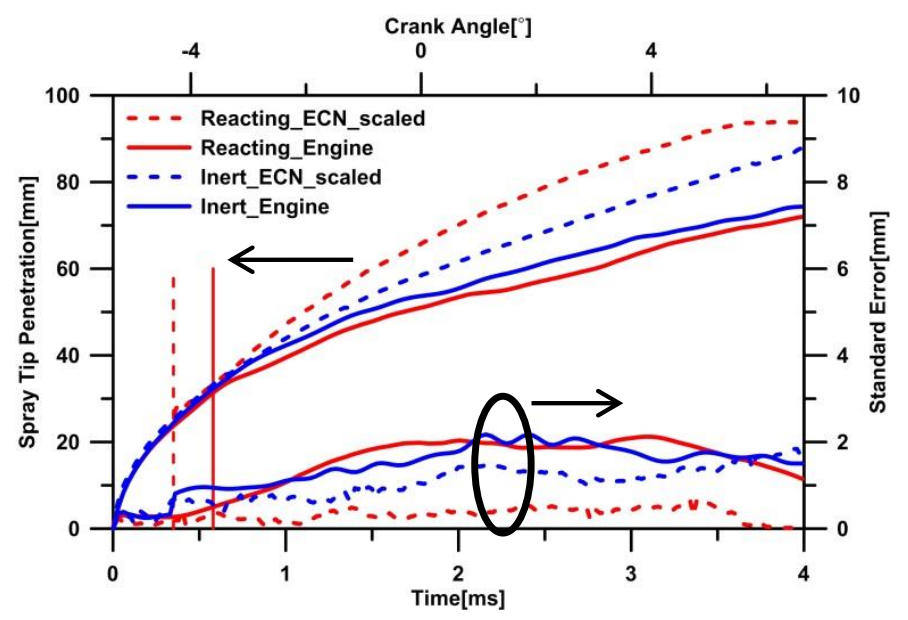

Figure 16.Comparison of tip penetration with ECN data [11] under reacting conditions at SA1500 point

The effects of TDC temperature on ignition delay and lift-off length were presented in Figure 17 and Figure 18 respectively. Note that the temperature sweep was carried out at constant TDC density, oxygen content and injection pressure. ECN data under the same ambient density, oxygen concentration and injection pressure was also shown here as a reference. There may be a nozzle diameter effect on ID, but there is no simple re-scaling as in the case of penetration, so no correction has been performed. As for LOL, ECN data have been scaled according to the empirical result from Siebers [5] [6]

$$
\mathrm{LOL}_{\text {scale }}=\mathrm{LOL} \cdot\left(\frac{\mathrm{d}_{0 \mathrm{ENG}}}{\mathrm{d}_{0 \mathrm{ECN}}}\right)^{0.34}
$$

Both ID and LOL exhibit a much lower sensitivity to in-cylinder temperature compared to results under quiescent conditions. It is remarkable that above $850 \mathrm{~K}$ of both parameters are almost constant. These results are difficult to explain with the present data. CFD analysis is underway to clarify if flow interaction could also have an influence on this result. On the other hand, while standard deviation of ID is pretty similar in both facilities, for LOL the standard deviation of the engine is much larger than that of quiescent data, which is consistent with previous work [20] and could stem from flow interaction.

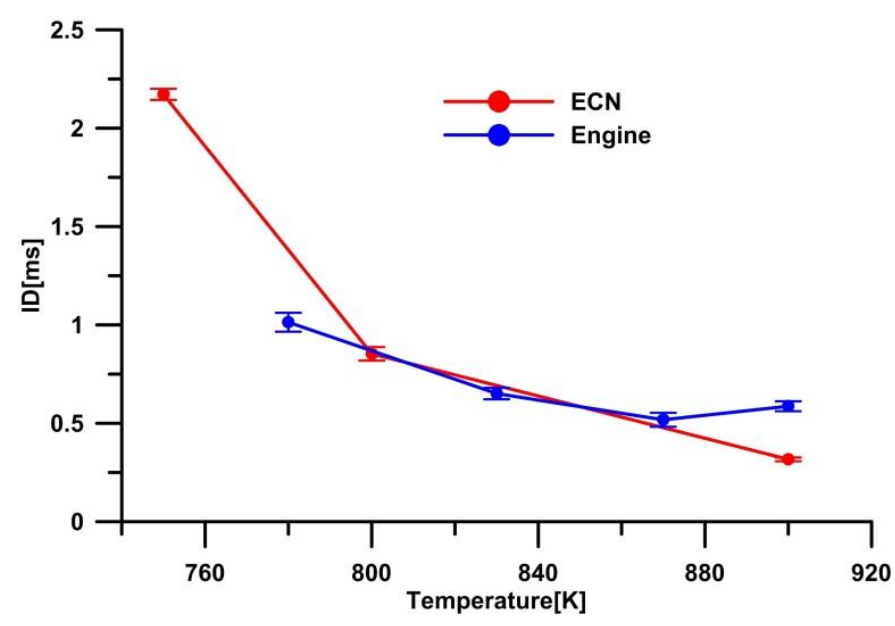

Figure 17.Ignition delay with temperature sweep $\left(\mathrm{P}_{\text {inj }}=1500 \mathrm{bar}, \mathrm{O}_{2}=21 \%\right.$, $\left.\rho_{\mathrm{a}}=22.8 \mathrm{Kg} / \mathrm{m} 3\right)$

Page 10 of 12

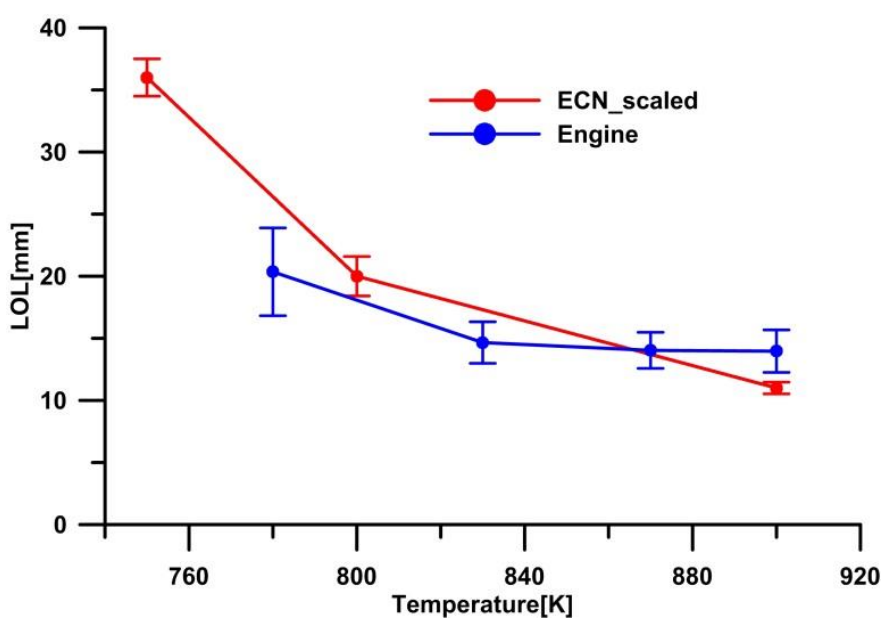

Figure 18. Lift-off Length with temperature sweep $\left(\mathrm{P}_{\mathrm{inj}}=1500 \mathrm{bar}, \mathrm{O}_{2}=21 \%\right.$, $\rho_{\mathrm{a}}=22.8 \mathrm{Kg} / \mathrm{m}^{3}$ )

Finally, the effects of TDC ambient temperature on spray tip penetration and corresponding SE were shown in Figure 19.It seems the temperature does not have significant influence on penetration (except SA1500) which is different from the observed trend under quiescent conditions [10][11] where the spray shows a faster penetration with higher temperature. In addition, SE shows a fast increase after ignition (ignition delay is shown in Figure 17 ) and it increases with higher temperature. This trend might be explained with similar ID and LOL (as shown in Figure 17 and Figure 18 ), which may contribute to a similar radial and axial expansion on spray under the same ambient density.

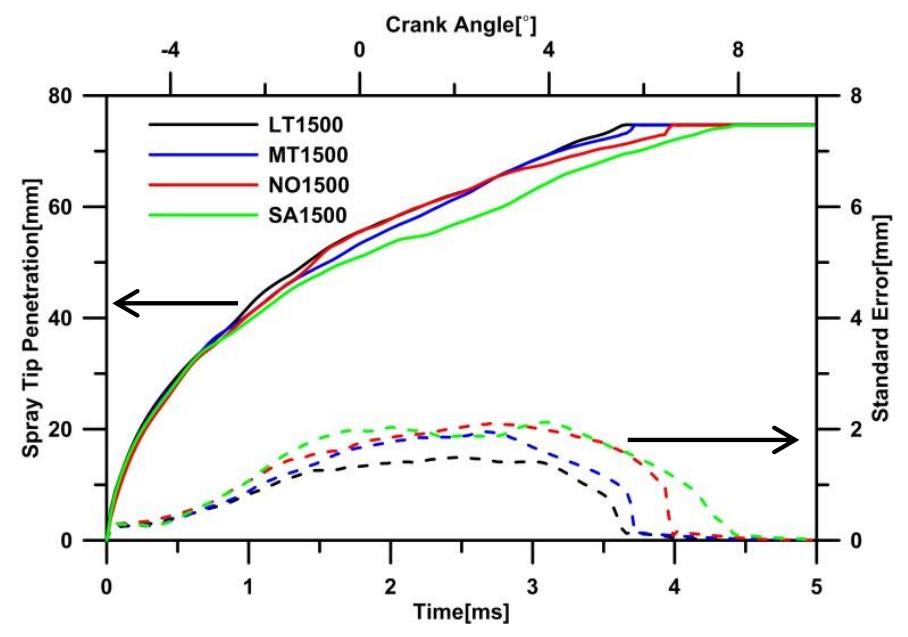

Figure 19. Tip penetration (solid lines) and corresponding standard error of the mean (dashed lines) with temperature sweep $\left(\rho_{\mathrm{a}}=22.8 \mathrm{Kg} / \mathrm{m}^{3}, \mathrm{P}_{\mathrm{inj}}=\right.$ 1500bar)

\section{Conclusions}

In this paper, the characteristics of diesel spray injected into a nonquiescent chamber under both non-reacting and reacting conditions with a single-hole injector were investigated. The liquid length, spray tip penetration, ignition delay and flame lift-off length under different thermodynamic conditions and injection pressure values were measured with different kinds of optical techniques. Some parameters were also compared with ECN data from a constant-pressure vessel 
where the ambient condition was considered as quiescent. In addition, in order to have a better understanding about the gas flow effects on spray behavior, the in-cylinder airflow velocity field was also measured with PIV technique. Some important conclusions of this study are summarized as follows:

- Before TDC, the airflow velocity at the lower part of the chamber was very high compared to the nozzle area. Some vortexes due to the reversing motion of the piston at the end of compression and start of expansion were found, which interacted with the injection event. Thanks to the measurement at different planes, it was found the velocity field in the combustion chamber was not symmetric.

- Under non-reacting conditions, Schlieren technique was not able to identify spray tip very well when it penetrated more than $50 \mathrm{~mm}$ because of the interference from airflow. A UV-LA technique was developed and it works better on capturing spray penetration under such non-quiescent condition when the spray becomes diluted and the Schlieren effect from the spray is not strong enough compared to the background.

- As for the non-reacting spray, the tip penetration from the engine was kept almost consistent with ECN data at the start of injection. When it came to the high velocity region of airflow (around 40mm), the spray was slowed down owning to the aerodynamic force and vortexes from airflow.

- $\quad$ As for the reacting spray, the divergence of tip penetration with the ECN data appears earlier than that of non-reacting spray. Both ignition delay and flame lift-off length from the engine show much less sensitivity with ambient temperature sweep. Ambient temperature did not have significant effect on spray tip penetration.

\section{References}

1. Naber, J. and Siebers, D., "Effects of Gas Density and Vaporization on Penetration and Dispersion of Diesel Sprays," SAE Technical Paper 960034, 1996, doi:10.4271/960034.

2. Pickett, L., Manin, J., Genzale, C., Siebers, D. et al., "Relationship Between Diesel Fuel Spray Vapor Penetration/Dispersion and Local Fuel Mixture Fraction," SAE Int. J. Engines 4(1):764-799, 2011, doi:10.4271/2011-01-0686.

3. Siebers, D., "Liquid-Phase Fuel Penetration in Diesel Sprays," SAE Technical Paper 980809, 1998, doi:10.4271/980809.

4. Siebers, D., "Scaling Liquid-Phase Fuel Penetration in Diesel Sprays Based on Mixing-Limited Vaporization," SAE Technical Paper 1999-01-0528, 1999, doi:10.4271/1999-01-0528.

5. Siebers, D. and Higgins, B., "Flame Lift-Off on Direct-Injection Diesel Sprays Under Quiescent Conditions," SAE Technical Paper 2001-01-0530, 2001, doi:10.4271/2001-01-0530.

6. Siebers, D., Higgins, B., and Pickett, L., "Flame Lift-Off on Direct-Injection Diesel Fuel Jets: Oxygen Concentration Effects," SAE Technical Paper 2002-01-0890, 2002, doi:10.4271/2002-01-0890.

7. Higgins, B., Siebers, D., and Aradi, A., "Diesel-Spray Ignition and Premixed-Burn Behavior," SAE Technical Paper 2000-010940, 2000, doi:10.4271/2000-01-0940.

8. Pickett, L., Siebers, D., and Idicheria, C., "Relationship Between Ignition Processes and the Lift-Off Length of Diesel Fuel Jets,"
SAE Technical Paper 2005-01-3843, 2005, doi:10.4271/200501-3843.

9. Pastor, J., Payri, R., Garcia-Oliver, J., and Briceño, F., "Schlieren Methodology for the Analysis of Transient Diesel Flame Evolution," SAE Int. J. Engines 6(3):1661-1676, 2013, doi:10.4271/2013-24-0041.

10. Desantes J. M., Pastor J. V., García-Oliver J. M., Briceño F. J., An experimental analysis on the evolution of the transient tip penetration in reacting Diesel sprays, Combustion and Flame, 161:8 (2014) 2137-2150

11. Payri R., García-Oliver J. M., Xuan T., M. Bardi, A study on diesel spray tip penetration and radial expansion under reacting conditions, Applied Thermal Engineering , 90 (2015) 619-629

12. Nerva J. G., An assessment of fuel physical and chemical properties in the combustion of a Diesel spray, $\mathrm{PhD}$ Thesis, 2013

13. Hult, J., Matlok, S., and Mayer, S., "Optical Diagnostics of Fuel Injection and Ignition in a Marine Two-Stroke Diesel Engine," SAE Int. J. Engines 7(3):1195-1206, 2014, doi:10.4271/2014-01-1448.

14. Pastor, J., Garcia-Oliver, J., Bermudez, V., and Micó, C., "Spray Characterization for Pure Fuel and Binary Blends under NonReacting Conditions," SAE Technical Paper 2014-01-1407, 2014, doi:10.4271/2014-01-1407.

15. Eagle, W., Malbec, L., and Musculus, M., "Measurements of Liquid Length, Vapor Penetration, Ignition Delay, and Flame Lift-Off Length for the Engine Combustion Network 'Spray B' in a 2.34 L Heavy-Duty Optical Diesel Engine," SAE Int. J. Engines 9(2):910-931, 2016, doi:10.4271/2016-01-0743.

16. Clément C., Ulf A., Öivind A, Rolf E., et al., Influence of jet-jet interactions on the lift-off length in an optical heavy-duty DI diesel engine, Fuel, 112 (2013) 311-318

17. R., Zhang, Y., Zhang, S, Kook., Morphological variations of inflame and exhaust soot particles associated with jet-to-jet variations and jet-jet interactions in a light-duty diesel engine, Combustion and Flame, 176 (2017) 377-390

18. Zha, K., Busch, S., Miles, P., Wijeyakulasuriya, S. et al., "Characterization of Flow Asymmetry During the Compression Stroke Using Swirl-Plane PIV in a Light-Duty Optical Diesel Engine with the Re-entrant Piston Bowl Geometry," SAE Int. J. Engines 8(4):1837-1855, 2015, doi:10.4271/2015-01-1699.

19. Perini, F., Zha, K., Busch, S., Miles, P. et al., "Principal Component Analysis and Study of Port-Induced Swirl Structures in a Light-Duty Optical Diesel Engine," SAE Technical Paper 2015-01-1696, 2015,

20. Payri, F., Pastor, J., Nerva, J., and Garcia-Oliver, J., "Lift-Off Length and KL Extinction Measurements of Biodiesel and Fischer-Tropsch Fuels under Quasi-Steady Diesel Engine Conditions," SAE Int. J. Engines 4(2):2278-2297, 2011, doi:10.4271/2011-24-0037.

21. Engine Combustion Network webpage: http://www.sandia.gov/ecn (online).

22. Bermúdez, V., García, J., Juliá, E., and Martínez, S., "Engine with Optically Accessible Cylinder Head: A Research Tool for Injection and Combustion Processes," SAE Technical Paper 2003-01-1110, 2003, doi:10.4271/2003-01-1110.

23. Settles G. S., Schlieren and sadowgraph techniques: visualizing phenomena in transparent media (Experimental Fluid Mechanic), Springer Verlag, Heidelberg, Germany, 2001

24. Benajes J., Payri R., Bardi M., Martí-Aldaraví P., Experimental characterization of diesel ignition and lift-off length using a single-hole ECN injector, Applied Thermal Engineering 58 (2013) 554-563

Page 11 of 12 
25. Yamakawa, M., Takaki, D., Li, T., Zhang, Y. et al., "Quantitative Measurement of Liquid and Vapor Phase Concentration Distributions in a D.I. Gasoline Spray by the Laser Absorption Scattering (LAS) Technique," SAE Technical Paper 2002-01-1644, 2002, doi:10.4271/2002-01-1644.

26. Zhao H. Laser Diagnostics and Optical Measurement

Techniques in Internal Combustion Engines. SAE International, Warrendale, 2012.

27. Reche C. M., Development of measurement and visualization techniques for characterization of mixing and combustion processes with surrogate fuels, PhD Thesis, 2015

28. "How Frame-Straddling works". Information available at http://www.tsi.com.

29. Sanghoon K., Pickett L.M., Liquid length and vapor penetration of conventional, Fischer-Tropsch, coal-derived, and surrogate fuel sprays at high-temperature and high-pressure ambient conditions, Fuel 93 (2012) 539-548

30. Payri R., García-Oliver J. M., Bardi M., Manin J., Fuel temperature influence on Diesel sprays in inert and reacting conditions, Applied Thermal Engineering 35 (2012) 185-195

31. John Abraham, Entrainment characteristics of transient gas jets, Numerical Heat Transfer, Part A: Applications Vol. 30, Iss.4, 1996

\section{ACKNOWLEDGMENTS}

This work was partially funded by the Government of Spain through COMEFF Project (TRA2014-59483-R). In addition, the authors acknowledge that some equipment used in this work has been partially supported by FEDER project funds (FEDER-ICTS-2012-06), framed in the operational program of unique scientific and technical infrastructure of the Ministry of Science and Innovation of Spain. The authors want also to express their gratitude to CONVERGENT SCIENCE Inc for their kind support for this research.

\section{DEFINITIONS/ABBREVIATIONS}

TDC

ECN

LOL

UV-LA

CMOS

PIV

ATDC

FWHM

ASOI

ASOE

$\mathbf{T}_{\mathbf{a}}$

$\rho_{\mathrm{a}}$

EVC

ID

SE top dead center

engine combustion

network

lift-off length

ultraviolet light

absorption

Complementary Metal

Oxide Silicon

particle image

velocimetry

after top dead center

full width at half

maximum

after start of injection

after start of energizing

Ambient temperature

Ambient denstiy

exhaust vent closure

ignition delay

Standard error 